\title{
Matrix Equivalence over Commutative Local Rings with Maximal Ideal Generated by Two 2-nilpotent Elements
}

\author{
Alex Yakyma \\ email: alex@yakyma.com
}

August 30, 2018

\begin{abstract}
In this paper the matrix equivalence problem is proven tame and solved for commutative local rings with 2-generated maximal ideal such that the square of each generator equals zero.
\end{abstract}

\section{Introduction}

Matrix equivalence over commutative rings is vital to a number of linear classification problems in algebra. The problem of matrix equivalence over a commutative ring is called wild if it contains the problem of classification of pairs of matrices up to simultaneous similarity over a field; otherwise it is called tame. Wild problems are believed to be "hopeless" and it is important to determine for which rings the problem of matrix equivalence is tame vs. wild. Some classic examples of tame matrix equivalence problems are the cases when the underlying ring is a principal ideal domain or a local ring of principal ideals (see [1]).

It is known [2], however, that the problem of matrix equivalence is wild over Noetherian unique factorization domains that are not a principle ideal domain and that it is wild over any local ring that contains a 3-generated ideal. An extension of that result is provided in [3], where it is proven that the problem is wild for local rings with 2-generated maximal ideal $J$ such that for some generators $u$ and $v$ of $\mathrm{J}, u^{2} \notin L v, u v \notin L u^{2}+L v^{2}$, $v^{2} \notin L u v$. In the same article it is shown that the problem is wild over some classes of Noetherian local rings with 2-generated maximal ideal (including the case when the ring is integrally closed).

In this paper the matrix equivalence problem is proven tame and solved for commutative local rings with 2-generated maximal ideal such that the square of each generator equals zero. This extends the previously achieved result for local rings with $J^{2}=0$ to a case where this condition is replaced by a weaker one: $J=L u+L v, u^{2}=v^{2}=0$ while $u v$ may not be equal to 0 . 


\section{The Main Result}

Throughout the paper, it is assumed that all rings are commutative and with unity. By $J(L)$ (or just $J$, for short) we denote the Jacobson radical of ring $L$.

The question of equivalence of $m \times n$-matrices over such rings will be considered: two $m \times n$-matrices $A, B$ are equivalent if there exist invertible matrices $C$ and $D$ of suitable dimensions such that $A C=D B$. The fact that $A$ is equivalent to $B$ is denoted by $A \sim$ $B$. Additionally, for an arbitrary ring $R$, pairs of matrices $\left(A, A^{\prime}\right)$ and $\left(B, B^{\prime}\right)$ are called simultaneously equivalent if there exist invertible matrices $C$ and $D$ such that $A C=D B$ and $A^{\prime} C=D B^{\prime}$. The fact that pairs $\left(A, A^{\prime}\right)$ and $\left(B, B^{\prime}\right)$ are simultaneously equivalent is denoted as $\left(A, A^{\prime}\right) \approx\left(B, B^{\prime}\right)$. Let $A$ be a matrix over $L$. Denote by $\operatorname{dim} A$ the $\operatorname{dimensions~of~}$ matrix $A$, i.e. a pair integers $(m, n)$.

The goal of this paper is proving the following result:

Theorem. Let $L$ be a local ring with 2-generated maximal ideal $J=L u+L v$ such that $u^{2}=$ $v^{2}=0$ and $u v \neq 0$. The matrix equivalence problem over $L$ is tame.

Later in the paper, the main result will be extended to specify the canonical form of every matrix over $L$.

\section{The Structure of the Proof}

The concept described in the definition below is used extensively throughout the article.

Definition. If $A$ is a matrix over a local ring $L$ such that all of the elements of $A$ are taken from the ideal $V$ then $A$ is called a $\boldsymbol{V}$-matrix. In particular, when $V=J$ is the maximal ideal of $L$, $A$ is called a $\boldsymbol{J}$-matrix.

The overall idea of the proof of the main theorem is to "split" the problem into different "layers" by powers of the maximal ideal $J$. So, it is proven that any matrix $A$ is equivalent to a block-diagonal matrix of the form:

$$
\left[\begin{array}{cc}
I & 0 \\
0 & A^{\prime}
\end{array}\right]
$$

where $A^{\prime}$ is a $J$-matrix and two matrices are equivalent iff the corresponding diagonal blocks are equivalent.

The process continues further, showing that $J$-matrices themselves have a certain type of block-diagonal reduction of the form:

$$
\left[\begin{array}{cc}
X & 0 \\
0 & A^{\prime \prime}
\end{array}\right]
$$


with $X$ being structurally identical to a canonical form of a pair of matrices over a field with respect to simultaneous equivalence and $A^{\prime \prime}$ being a $J^{2}$-matrix. And similarly as before, two $J$-matrices are equivalent when the corresponding diagonal blocks are equivalent.

And finally, $A^{\prime \prime}$ gets reduced to a matrix:

$$
\left[\begin{array}{cc}
I u v & 0 \\
0 & A^{(3)}
\end{array}\right]
$$

where $A^{(3)}$ is a $J^{3}$-matrix and therefore is a zero-matrix and two matrices are equivalent when the corresponding diagonal blocks are equivalent.

From the conceptual standpoint, the essential part of the proof is the idea of "lifting" a certain type of simpler form from that over a factor ring $L / J^{n}$ to that over $L / J^{n-1}$. The most laborious portion turns out to be the lifting process from the simplified form of $J$-matrices modulo $J^{2}$ to a form of a $J$-matrix over ring $L$. This is due to the relative complexity of the canonical form of pairs of matrices compared to the case of single matrices.

So, in summary, there are three major steps that add a corresponding matrix equivalence problem over field $F=L / J$ to the stack:

1) Equivalence of any matrices over $L / J$ being lifted to $L / J^{2}$; it adds the problem of equivalence of single matrices over $F$

2) Equivalence of $J$-matrices over $L / J^{2}$ being lifted to $L / J^{3}$; adds the problem of simultaneous equivalence of pairs of matrices over $F$

3) Equivalence of $J^{2}$-matrices over $L / J^{3} \cong L$ is itself contained in the problem of equivalence of single matrices over $F$.

\section{Preliminaries}

Let $A$ be a matrix over local ring $L$ with maximal ideal $J$. It will be assumed, by definition, that $\operatorname{rank} A=\operatorname{rank} \bar{A}$ where $\bar{A}$ is a corresponding matrix over field $L / J$.

The following two lemmas provide important insight into the modular structure of ideals of factor rings that will be needed later in the article.

Lemma 1. Let $M$ be a module over local ring $L$ generated by $n$ elements: $M=L a_{1}+\cdots+L a_{n}$. If $M$ can be generated by a smaller number of elements $m$, then those elements can be selected from among $a_{1}, \ldots, a_{n}$.

Proof. Let $L=L u_{1}+\cdots+L u_{m}, m<n$. Then there exists an $n \times m$-matrix $U$ such that $\vec{a}=U \vec{u}$, where $\vec{a}=\left[a_{1}, \ldots, a_{n}\right]^{\top}$ and $\vec{u}=\left[u_{1}, \ldots, u_{m}\right]^{\top}$. But since $a_{1}, \ldots, a_{n}$ also generate $M$, there exists an $m \times n$-matrix $V$ such that $\vec{u}=V \vec{a}$. Combining the two equalities implies that $\left(I_{n}-U V\right) \vec{a}=0$. Since for any two matrices $A$ and $B, \operatorname{rank} A B \leqslant \min (\operatorname{rank} A, \operatorname{rank} B)$, $\operatorname{rank} U V \leqslant m<n$. But then $\operatorname{rank}\left(I_{n}-U V\right) \geqslant 1$ and thus there exists $i(1 \leqslant i \leqslant n)$ such that $a_{i}=\sum_{i=1, j \neq i}^{n} \xi_{j} a_{j}$, which implies that $a_{i}$ can be excluded from the set of generators of $M$. 
Applying the same logic recursively to the remaining set of generators, the process will stop when the set contains exactly $m$ elements $a_{i_{1}}, \ldots, a_{i_{m}}$ such that $M=L a_{i_{1}}+\cdots+L a_{i_{m}}$.

Lemma 2. Let $L$ be a local ring with maximal ideal J. If $J^{n}(n \geqslant 1)$ is an m-generated ideal then $J^{n} / J^{n+1}$ is an m-generated L-module that is isomorphic to a direct sum of $m$ instances of field $F=L / J$.

Proof. Let $J^{n}$ be $m$-generated and $J^{n}=L u_{1}+\cdots+L u_{m}$. Then clearly $J^{n} / J^{n+1}$ is generated by $\bar{u}_{1}, \ldots, \bar{u}_{m}$. Suppose that $J^{n} / J^{n+1}$ can be generated by $m-1$ elements. Then according to lemma 1, a set of $m-1$ generators can be selected from among $\bar{u}_{1}, \ldots, \bar{u}_{m}$. Without loss of generality, assume $J^{n} / J^{n+1}$ is generated by $\bar{u}_{1}, \ldots, \bar{u}_{m-1}$. Then there exist such $a_{i}$ that $u_{m}-a_{1} u_{1}-\cdots-a_{m-1} u_{m-1}=a \in J^{n+1}$. By definition, $a=\sum_{i=1}^{m} b_{i} u_{i}$, with some $b_{i} \in J$. But then $u_{m}=\sum_{i=1}^{m-1}\left(1-b_{m}\right)^{-1}\left(a_{i}+b_{i}\right) u_{i}$, which is a contradiction, therefore proving that $J^{n} / J^{n+1}$ is $m$-generated.

Suppose now that there exists a non-zero element $\bar{x} \in \bar{L} \bar{u}_{i} \cap \sum_{k=1, k \neq i}^{m} \bar{L} \bar{u}_{k}$ for some fixed $i$. Then

$$
\bar{x}=\bar{l}_{i} \bar{u}_{i}=\sum_{k=1, k \neq i}^{m} \bar{l}_{k} \bar{u}_{k}
$$

where $l_{i} \in L^{*}$ and $l_{k} \in L, k \neq i$. Equality 1 implies that $l_{i} u_{i}=\sum_{k=1, k \neq i}^{m} l_{k} u_{k}+\sum_{k=1}^{m} a_{k} u_{k}$, $a_{k} \in J$. But this means that $u_{i}=\sum_{k=1, k \neq i}^{m}\left(l_{i}-a_{i}\right)^{-1}\left(l_{k}+a_{k}\right) u_{k}$, which is not possible since $J^{n} / J^{n+1}$ is $m$-generated. This implies that for any $i$, submodules $\bar{L} \bar{u}_{i}$ and $\sum_{k=1, k \neq i}^{m} \bar{L} \bar{u}_{k}$ of $J^{n} / J^{n+1}$ have only zero intersection and at the same time, $J^{n} / J^{n+1}=\sum_{k=1}^{m} \bar{L}_{k}$. The latter means that $J^{n} / J^{n+1}=\bigoplus_{i=1}^{m} \bar{L} \bar{u}_{i}$.

Fix $i(1 \leqslant i \leqslant m)$ and consider natural module homomorphism $\psi_{i}: L \rightarrow \bar{L} \bar{u}_{i}$ by setting $\psi_{i}(l)=\bar{l} \bar{u}_{i}$ for any $l \in L$. But then clearly $\operatorname{Ker} \psi_{i}=J$ and $\bar{L} \bar{u}_{i} \cong F$ as $L$-modules. Lemma is proven.

As it has been proven in [4], every matrix over $L$ is equivalent to a matrix of the form $\operatorname{diag}\left[I_{k}, A^{\prime}\right]$ where $A^{\prime}$ is a $J$-matrix. Moreover, two matrices are equivalent if and only if the identity matrices in such block-diagonal form are of the same dimension and corresponding $J$-matrices are equivalent, thus reducing the question of matrix equivalence to the equivalence of $J$-matrices.

The following notation will be used throughout this paper:

$$
P_{n}=\left[\begin{array}{ccccc}
v & u & & & \\
& v & u & & \\
& & \ddots & \ddots & \\
& & & v & u
\end{array}\right]
$$




$$
\begin{gathered}
Q_{n}=\left[\begin{array}{llll}
v & & & \\
u & v & & \\
& u & \ddots & \\
& & \ddots & v \\
& & & u
\end{array}\right] \\
R_{n}=\left[\begin{array}{cccc}
u & v & & \\
& \ddots & \ddots & \\
& & u & v \\
& & & u
\end{array}\right] \\
u \\
u \\
v
\end{gathered}
$$

where $P_{n}$ is an $n \times(n+1)$-matrix, $Q_{n}$ is an $(n+1) \times n$-matrix, $R_{n}, S_{n}(\vec{\alpha})$ are $n \times n$-matrices, $n \geqslant 1, \vec{\alpha}=\left(\alpha_{1}, \ldots, \alpha_{n}\right)$ with $\alpha_{1}, \ldots, \alpha_{n} \in L$, and all the rest of the elements of the matrices are zeros. Matrices $P$ and $R$ have only two non-zero diagonals: the main one and the one above it. Matrix $Q$ also has only two non-zero diagonals: the main one and the one below it. Matrix $S$ has two non-zero diagonals (the main one and the one above it) and, in the general case, the bottom row is non-zero.

For the rest of the article $L$ will be a local ring with 2-generated maximal ideal $J=$ $L u+L v, u^{2}=v^{2}=0$ and $u v \neq 0$.

The next lemma underlines the structural properties of $J$-matrices with respect to equivalence modulo $J^{2}$.

Lemma 3. Let $A$ be a J-matrix. Then $A$ is equivalent modulo Luv to a matrix of the form:

$$
\left[\begin{array}{cccc}
X_{1} & & & \\
& \ddots & & \\
& & X_{r} & \\
& & & 0
\end{array}\right]
$$

where each $X_{i}$ is one of the $P, Q, R$ or S-blocks of some dimensions, all the rest of the elements are zeros and the zero matrix down the main diagonal has dimension $\geqslant 0$. The block-diagonal matrix 2 is uniquely determined by matrix A: A can be equivalent to only one such matrix modulo Luv up to permutation of blocks. 
Proof. According to lemma 2, $J / J^{2}=\bar{L} \bar{u} \oplus \bar{L} \bar{v}$ and $\bar{L} \bar{u} \cong \bar{L} \bar{v} \cong F$ as $L$-modules. But that implies that for any $J$-matrices $A=A_{1} u+A_{2} v$ and $B=B_{1} u+B_{2} v, A \sim B\left(\bmod J^{2}\right)$ is equivalent to the fact that $\left(A_{1}, A_{2}\right) \approx\left(B_{1}, B_{2}\right)(\bmod J)$. Now, applying the generic KroneckerWeierstrass form (from [5]) to the corresponding matrix pencils and remembering that $J^{2}=L u v$, concludes the proof of the lemma.

The following two lemmas will be very useful for elementary transformations of $J$ matrices. They demonstrate an interesting property of rings with condition $u^{2}=v^{2}=0$.

Lemma 4. Let $t=\alpha u+\beta v \in J$, where $\alpha, \beta \in L^{*} \cup\{0\},\{\alpha, \beta\} \neq\{0\}$. Then for any $a, b \in J^{2}$ there exists $\omega \in L^{*}$ such that $\omega \equiv 1(\bmod J)$ and $(t+a) \omega=t+b$.

Proof. Set $a=a_{1} u v, b=b_{1} u v, a_{1}, b_{1} \in L$. Also, let $\mu$ be a non-zero element of the set $\{\alpha, \beta\}$. Define a map: $f:\{\alpha, \beta\} \rightarrow\{u, v\}$ as follows: $f(\alpha)=v$ and $f(\beta)=u$. Set $\omega=1+\mu^{-1} f(\mu)\left(b_{1}-\right.$ $\left.a_{1}\right)$. Then clearly $\omega \equiv 1(\bmod J)$ and $(t+a) \omega=t+b$.

Lemma 5. Let $t=\alpha u+\beta v \in J$, where $\alpha, \beta \in L^{*} \cup\{0\},\{\alpha, \beta\} \neq\{0\}$. Then for any $a, b \in J^{2}$ there exists $\omega \in L$ such that $b-(t+a) \omega=0$.

Proof. Let $b=b_{1} u v, b_{1} \in L$ and let $\mu$ be a non-zero element of the set $\{\alpha, \beta\}$. Similarly as before, define a map: $f:\{\alpha, \beta\} \rightarrow\{u, v\}$ by setting: $f(\alpha)=v$ and $f(\beta)=u$. Then setting $\omega=\mu^{-1} f(\mu) b_{1}$ provides the desired condition.

Having done the required preparation, the next objective will be to prove a consecutive set of statements regarding different block types (lemmas 6-9).

Lemma 6. Let $A$ be a J-matrix equivalent modulo $J^{2}$ to

$$
\left[\begin{array}{cc}
P_{n} & 0 \\
0 & A^{\prime}
\end{array}\right]
$$

where $A^{\prime}$ is a J-matrix. Then $A$ is equivalent to

$$
\left[\begin{array}{cc}
P_{n} & 0 \\
0 & \tilde{A}
\end{array}\right]
$$

where $\tilde{A} \equiv A^{\prime}\left(\bmod J^{2}\right)$.

Note that what lemma 6 actually states is that equivalence to the block-diagonal matrix can be "lifted" from equivalence modulo $J^{2}$ to equivalence over $L$ and the only impact of such "lifting" process is contained within the right lower block and is irrelevant modulo $J^{2}$. Similar observation applies to analogous lemmas further in the article with respect to other block types. 
Proof. According to the conditions of the lemma, $A$ is equivalent to a matrix $A_{1}$ of the form:

$$
\left[\begin{array}{ccccc|c}
v+* & u+* & * & \cdots & * & \\
* & v+* & u+* & & & \\
\vdots & * & \ddots & \ddots & * & * \\
* & & & v+* & u+* & \\
\hline & & & & & \\
& & * & & & A^{\prime}
\end{array}\right]
$$

where by $*$ we denote some elements from $J^{2}$.

According to lemma 5, any * element in position $(i, j)$, where $1 \leqslant i \leqslant n$ and $j \notin\{i, i+1\}$, can be nulled by multiplying column $i$ by corresponding $\omega \in J$ and subtracting it from column $j$. Such an operation influences the rest of the rows as follows: a) if $i>1$ then the elements of row $i-1$ will have updated $*$ values and $b$ ) for any $i$, the elements of matrix $A^{\prime}$ will likewise obtain some new * addendums; note however that none of these changes are relevant modulo $J^{2}$ and therefore do not alter the overall structure of the matrix. The initial objective is to eliminate all $*$ in the first $n$ rows of matrix $A_{1}$. The process starts at row $n$, where with the help of column $n$, all the $*$ values are nulled in the row except in positions $(n, n)$ and $(n, n+1)$. The matrix then acquires a new form $\left(A_{2}\right)$ :

$$
\left[\begin{array}{ccccc|ccc}
v+* & u+* & * & \ldots & * & & & \\
* & v+* & u+* & & & & & \\
\vdots & * & \ddots & \ddots & * & & * & \\
0 & \ldots & 0 & v+* & u+* & 0 & \ldots & 0 \\
\hline & & * & & & & A^{\prime \prime} &
\end{array}\right]
$$

The next step involves the same operation but this time with row $n-1$, then row $n-2$ and so on until row 1 is processed. Note that at every one of these steps, say the step for row $n-k$, the previously processed rows (the ones from $n-k+1$ through $n$ ) are not affected at all. The process ends when the matrix is equivalent to $A_{3}$ :

$$
\left[\begin{array}{ccccc|c}
v+* & u+* & 0 & \cdots & 0 & \\
0 & v+* & u+* & & & \\
\vdots & \mathbf{0} & \ddots & \ddots & \mathbf{0} & \mathbf{0} \\
0 & & & v+* & u+* & \\
\hline & & & & & A^{(3)}
\end{array}\right]
$$


The next objective is getting rid of $*$ in the left lower block of $A_{3}$. That will be achieved in two stages. At stage one, analogous steps will be taken as previously, only this time applied to columns instead of rows: the element of the main diagonal will be used to null the $*$ in the entire column (by multiplying the $i$-th row by a suitable element $\omega$ and subtracting it from the target row until the entire column $i$ is processed). This time, however, the process starts with column 1 and finishes at column $n$. Note that every such step (say, step $j, j<n$ ) produces the following effect on other columns: it may introduce or update $*$ elements starting from position $(j, j+1)$ and through $(m, j)$ where $m$ is the number of rows in $A$. This effect however is easily addressed by the step for the next value of $j$. Thus the matrix ends up in the form $A_{4}$ :

$$
\left[\begin{array}{ccccc|c}
v+* & u+* & 0 & \cdots & 0 & \\
0 & v+* & u+* & & & \\
\vdots & \mathbf{0} & \ddots & \ddots & \mathbf{0} & \mathbf{0} \\
0 & & & v+* & u+* & \\
\hline & & & & * & \\
& & \mathbf{0} & & \vdots & A^{(3)}
\end{array}\right]
$$

where column $n+1$ will require further treatment.

Stage two is to get rid of the remaining * in the left lower block. For that, it is important to remember that in order to null those $J^{2}=L u v$ elements, it suffices to multiply row $n$ by $\omega$ values of the form $\theta u$, where $\theta \in L^{*} \cup\{0\}$. But multiplying $v+*$ by $\theta v$ gives zero and therefore these operations will not alter any 0 elements in the $n$-th column of the left lower block, thus reducing the matrix to $A_{5}$ :

$$
\left[\begin{array}{ccccc|c}
v+* & u+* & 0 & \cdots & 0 & \\
0 & v+* & u+* & & & \\
\vdots & \mathbf{0} & \ddots & \ddots & \mathbf{0} & \mathbf{0} \\
0 & & & v+* & u+* & \\
\hline & & \mathbf{0} & & & A^{(3)}
\end{array}\right]
$$

All that remains at this point is to remove $*$ from elements $v+*$ and $u+*$. Lemma 4 will be instrumental in this process. Namely, row 1 gets first multiplied by suitable $\omega \in L^{*}, \omega \equiv 1(\bmod J)$ so as to transform $v+*$ in position $(1,1)$ into $v$. This will affect the element at position $(1,2)$ but without changing its structure: it still remains an element of the form $u+*$. The next step is multiplying column 2 by some $\omega$ and thus reducing corresponding $u+*$ to $u$. Further repeating the steps in this manner (row $i$, column $i+1$ ), the process will finish on matrix $A_{6}$ of the form: 


$$
\left[\begin{array}{ccccc|c}
v & u & 0 & \ldots & 0 & \\
0 & v & u & & & \\
\vdots & \mathbf{0} & \ddots & \ddots & \mathbf{0} & \mathbf{0} \\
0 & & & v & u & \\
\hline & & \mathbf{0} & & & A^{(3)}
\end{array}\right]
$$

thus proving the lemma.

Lemma 7. Let $A$ be a J-matrix equivalent modulo $J^{2}$ to

$$
\left[\begin{array}{cc}
Q_{n} & 0 \\
0 & A^{\prime}
\end{array}\right]
$$

where $A^{\prime}$ is a J-matrix. Then $A$ is equivalent to

$$
\left[\begin{array}{cc}
Q_{n} & 0 \\
0 & \tilde{A}
\end{array}\right]
$$

where $\tilde{A} \equiv A^{\prime}\left(\bmod J^{2}\right)$.

Proof. The proof of the lemma is identical to the case of lemma 6 with the only difference of all operations being transposed, as $Q$-block is obviously a transposed version of the P-block.

Lemma 8. Let $A$ be a J-matrix equivalent modulo $J^{2}$ to

$$
\left[\begin{array}{cc}
R_{n} & 0 \\
0 & A^{\prime}
\end{array}\right]
$$

where $A^{\prime}$ is a J-matrix. Then $A$ is equivalent to

$$
\left[\begin{array}{cc}
R_{n} & 0 \\
0 & \tilde{A}
\end{array}\right]
$$

where $\tilde{A} \equiv A^{\prime}\left(\bmod J^{2}\right)$.

Proof. The proof of the lemma follows the proof of lemma 6 with a single modification: stage two is not required in the second part of the transformation as the left lower block gets entirely nulled via applying stage one process. 
Lemma 9. Let $A$ be a J-matrix equivalent modulo $J^{2}$ to

$$
\left[\begin{array}{cc}
S_{n}(\vec{\alpha}) & 0 \\
0 & A^{\prime}
\end{array}\right]
$$

where $A^{\prime}$ is a J-matrix. Then $A$ is equivalent to

$$
\left[\begin{array}{cc}
S_{n}(\vec{\alpha}) & 0 \\
0 & \tilde{A}
\end{array}\right]
$$

where $\tilde{A} \equiv A^{\prime}\left(\bmod J^{2}\right)$.

Proof. According to the conditions of the lemma, $A$ is equivalent to some matrix $A_{1}$ of the form:

$$
\left[\begin{array}{ccccc|c}
v+* & u+* & * & \ldots & * & \\
* & v+* & u+* & & & \\
\vdots & * & \ddots & \ddots & * & * \\
* & & & v+* & u+* & \\
\alpha_{1} u+* & \alpha_{2} u+* & & \alpha_{n-1} u+* & \alpha_{n} u+v+* & \\
\hline & & * & & & A^{\prime}
\end{array}\right]
$$

with $*$ being some elements from $J^{2}$. It is easy to see that by applying the same process as in the proof of lemmas 6, 8 (i.e. move row-by-row from $n$ to 1 using the elements of the main diagonal, according to lemma 5 , to eliminate corresponding $*$ values), $A_{1}$ can be transformed into $A_{2}$ of the following form:

$$
\left[\begin{array}{ccccc|c}
v+* & u+* & 0 & \cdots & 0 & \\
0 & v+* & u+* & & & \\
\vdots & \mathbf{0} & \ddots & \ddots & \mathbf{0} & \mathbf{0} \\
0 & & & v+* & u+* & \\
\alpha_{1} u+* & \alpha_{2} u+* & & \alpha_{n-1} u+* & \alpha_{n} u+v+* & \\
\hline & & * & & & A^{\prime \prime}
\end{array}\right]
$$

Next, nulling the left lower block of $A_{2}$ is the exact same two-stage process as in the proof of lemma 6 . Indeed, the upper $n-1$ rows of $S_{n}$ constitute a $P_{n-1}$-block and thus $A_{2}$ is equivalent to $A_{3}$ : 


$$
\left[\begin{array}{ccccc|c}
v+* & u+* & 0 & \cdots & 0 & \\
0 & v+* & u+* & & & \\
\vdots & \mathbf{0} & \ddots & \ddots & \mathbf{0} & \mathbf{0} \\
0 & & & v+* & u+* & \\
\alpha_{1} u+* & \alpha_{2} u+* & & \alpha_{n-1} u+* & \alpha_{n} u+v+* & \\
\hline & & 0 & & A^{\prime \prime}
\end{array}\right]
$$

Applying the same process as in the proof of lemma 6 to the upper $n-1$ rows (i.e. sequentially multiplying row 1 and then column 2 , row 3 , column 4 , etc. by proper invertible elements) results in matrix $A_{4}$ :

$\left[\begin{array}{ccccc|c}v & u & 0 & \ldots & 0 & \\ 0 & v & u & & & \\ \vdots & \mathbf{0} & \ddots & \ddots & \mathbf{0} & \mathbf{0} \\ 0 & & & v & u & \\ \alpha_{1} u+* & \alpha_{2} u+* & & \alpha_{n-1} u+* & \alpha_{n} u+v+* & \\ \hline & & & & & A^{\prime \prime}\end{array}\right]$

Applying lemma 5 * can be eliminated in elements at positions $(n, 1)$ through $(n, n-1)$ using the elements of the main diagonal, moving in the process from left to right. Finally, to null the remaining $*$ at position $(n, n)$, row $n-1$ is used, multiplied by $\theta v$, with some $\theta \in L^{*} \cup\{0\}$ and since $\theta v \cdot v=0$, the element in position $(n, n-1)$ will not be affected. Thus $A_{4}$ is reduced to $A_{5}$ : 


$$
\left[\begin{array}{ccccc|c}
v & u & 0 & \ldots & 0 & \\
0 & v & u & & & \\
\vdots & \mathbf{0} & \ddots & \ddots & \mathbf{0} & \mathbf{0} \\
0 & & & v & u & \\
\alpha_{1} u & \alpha_{2} u & & \alpha_{n-1} u & \alpha_{n} u+v & \\
\hline & & \mathbf{0} & & & A^{\prime \prime}
\end{array}\right]
$$

Lemma is proven.

The previous lemma concludes the series of statements about lifting canonical blocks from $L / J^{2}$ to $L$. That being said, however, an additional concept of "affined" blocks, and the two lemmas about their properties, will be needed to effectively describe the relationships between the $S$-blocks of different matrices.

Definition. Two blocks $S_{n}(\vec{\alpha})$ and $S_{n}(\vec{\beta})$ with $\vec{\alpha}=\left(\alpha_{1}, \ldots, \alpha_{n}\right)$ and $\vec{\beta}=\left(\beta_{1}, \ldots, \beta_{n}\right), \alpha_{i}, \beta_{i} \in$ $L, 1 \leqslant i \leqslant n$ are called affined (and denoted $S_{n}(\vec{\alpha}) \triangleq S_{n}(\vec{\beta})$ ) if $\alpha_{i} \equiv \beta_{i}(\bmod$ Lv) for all $i: 1 \leqslant$ $i \leqslant n$.

Lemma 10. Two blocks $S_{n}(\vec{\alpha})$ and $S_{n}(\vec{\beta})$ are affined iff $S_{n}(\vec{\alpha}) \equiv S_{n}(\vec{\beta})\left(\bmod J^{2}\right)$.

Proof. The proof easily follows from the fact that for any given $\mu \in\{0,1\}$, condition $x u+$ $\mu v \equiv y u+\mu v\left(\bmod J^{2}\right)$ is equivalent to $x \equiv y(\bmod L v)$.

Lemma 11. Two blocks $S_{n}(\vec{\alpha})$ and $S_{n}(\vec{\beta})$ are affined iff they are equivalent.

Proof. Let $S_{n}(\vec{\alpha}) \sim S_{n}(\vec{\beta})$. Then also $S_{n}(\vec{\alpha}) \sim S_{n}(\vec{\beta})\left(\bmod J^{2}\right)$ and according to lemma 3 . $S_{n}(\vec{\alpha}) \equiv S_{n}(\vec{\beta})\left(\bmod J^{2}\right)$. Then according to lemma $10, S_{n}(\vec{\alpha})$ and $S_{n}(\vec{\beta})$ are affined.

Conversely, assume $S_{n}(\vec{\alpha})$ and $S_{n}(\vec{\beta})$ are affined. Then for every $i: 1 \leqslant i \leqslant n, \beta_{i} u=$ $\alpha_{i} u+*$ where $* \in J^{2}$. But then applying the exact same process as in the final pass of proof of lemma 9 (i.e. applying lemma 5 and using the elements of the main diagonal and then finally the element at $(n-1, n)), S_{n}(\vec{\beta})$ can be transformed into $S_{n}(\vec{\alpha})$.

Following is the summary lifting statement from the equivalence modulo $J^{2}$ to the equivalence over ring $L$ itself.

Lemma 12. Let $A$ be a J-matrix. Then $A$ is equivalent to a matrix of the form: 


$$
\left[\begin{array}{lllll}
X_{1} & & & & \\
& X_{2} & & & \\
& & \ddots & & \\
& & & X_{r} & \\
& & & & A^{\prime}
\end{array}\right]
$$

where $X_{i}$ can be any of the blocks $P, Q, R$ or $S, A^{\prime}$ is a $J^{2}$-matrix and all the rest of the matrix elements are zeros. All $P, Q$ and $R$ blocks are uniquely determined by matrix A; S-blocks are unique modulo $J^{2}$; dimensions of $A^{\prime}$ are uniquely determined by $A$.

Proof. The proof directly follows from lemmas 3, 6, 7, 8 and 9 .

Now is the appropriate time to introduce the corresponding grouping process for blocks of different types.

Definition. Let $A$ be a J-matrix. Representation of the form 3 is called the fundamental form of $A$ and denoted as $\Phi(A)$. Note that a matrix can have multiple fundamental forms, so $\Phi$ should be thought of as a map that selects a particular matrix from the whole set of fundamental forms. Let $T=\Phi(A)$ is a matrix of the form 3 Denote by $\mathfrak{x}(T)$ the multiset consisting of all $P$-blocks of $T$, by $\mathfrak{Q}(T)$ the multiset of $Q$-blocks, by $\mathfrak{R}(T)$ the multiset of $R$-blocks and by $\mathfrak{S}(T)$ the multiset of $S$-blocks. $\mathfrak{I}^{(2)}(T)$ will denote matrix $A^{\prime}$.

Note that the reason why multisets are necessary in the definition above is due to the fact that $T$ may have multiple copies of identical blocks.

To be able to decompose the problem of equivalence of $J$-matrices into a set of independent problems, the following lemma will be used.

Lemma 13. Let $X$ be any of the blocks $P, Q, R$ or $S$ and $C, D$ are square matrices over $L$. If $X C$ is a $J^{2}$-matrix then $C \equiv 0(\bmod J)$. If $D X$ is a $J^{2}$-matrix then $D \equiv 0(\bmod J)$.

Proof. The proof is split by the block type.

Case 1: P-block. Consider product $Y=P_{n} C, C=\left[c_{i j}\right]$. Clearly, $Y=\left[v c_{i j}+u c_{i+1, j}\right]$. Since $Y \equiv 0\left(\bmod J^{2}\right)$, this implies that for every $i$ and $j, c_{i, j} \in J$.

Let $Z=\left[z_{i j}\right]=D P_{n}$. Then

$$
z_{i j}= \begin{cases}d_{i 1} v, & \text { for } j=1 \\ d_{i, j-1} u+d_{i j} v, & \text { for } 2 \leqslant j \leqslant n-1 \\ d_{i, n} u, & \text { for } j=n\end{cases}
$$

But then every element $d_{i j}$ participates in at least one of the three expressions that define $z_{i j}$ in 4 . This implies that all such elements $d_{i j}$ are in $J$.

Case 2: Q-block. It follows obviously, remembering that $Q$-block is a transposed version of a $P$-block. 
Case 3: R-block. Set $Y=R_{n} C, C=\left[c_{i j}\right]$. Then

$$
y_{i j}= \begin{cases}u c_{i j}+v c_{i+1, j}, & \text { for } 1 \leqslant i \leqslant n-1 \\ u c_{n j}, & \text { for } i=n\end{cases}
$$

Clearly, every $c_{i j}$ is part of at least one expression and therefore they are all in $J$.

Now set $Z=D R_{n}, D=\left[d_{i j}\right]$. Then

$$
z_{i j}= \begin{cases}d_{i 1} u, & \text { for } j=1 \\ d_{i, j-1} v+d_{i j} u, & \text { for } 2 \leqslant j \leqslant n\end{cases}
$$

and since all $d_{i j}$ are included, they all belong in $J$.

Case 4: S-block. Set $Y=S_{n}(\vec{\alpha}) C, C=\left[c_{i j}\right]$. Then

$$
y_{i j}= \begin{cases}v c_{i j}+u c_{i+1, j}, & \text { for } 1 \leqslant i \leqslant n-1 \\ \sum_{k=1}^{n-1} \alpha_{k} u c_{k j}+\left(\alpha_{n} u+v\right) c_{n j}, & \text { for } i=n\end{cases}
$$

All $c_{i j}$ participate in the first expression of 5 which implies that for all $i$ and $j, c_{i j} \equiv 0$ $(\bmod J)$.

Finally, for $Z=D S_{n}(\vec{\alpha})$ :

$$
z_{i j}= \begin{cases}d_{i 1} v+d_{i n} \alpha u, & \text { for } j=1 \\ \left(d_{i, j-1}+d_{i n} \alpha_{j}\right) u+d_{i j} v, & \text { for } 2 \leqslant j \leqslant n-1 \\ \left(d_{i, n-1}+d_{i n} \alpha_{n}\right) u+d_{i n} v, & \text { for } j=n\end{cases}
$$

All three expressions in 6 warrant $d_{i j} \in J$ for all $i$ and $j$.

Lemma is proven.

Now the desired decomposition can be approached.

Lemma 14. Let $A$ and $B$ be two J-matrices. $\tilde{A}=\Phi(A)$ and $\tilde{B}=\Phi(B)$ are fundamental forms of $A$ and $B$ respectively. Then $A \sim B$ iff the following conditions hold:

a) $\mathfrak{P}(\tilde{A})=\mathfrak{P}(\tilde{B}), \mathfrak{Q}(\tilde{A})=\mathfrak{Q}(\tilde{B}), \mathfrak{R}(\tilde{A})=\mathfrak{R}(\tilde{B})$

b) There exists a bijection $\tau: \mathfrak{S}(\tilde{A}) \rightarrow \mathfrak{S}(\tilde{B})$ such that for every $X \in \mathfrak{S}(\tilde{A}), \tau(X) \triangleq X$

c) $\mathfrak{I}^{(2)}(\tilde{A}) \sim \mathfrak{I}^{(2)}(\tilde{B})$.

Proof. Let $A \sim B$. Then according to lemma 12, conditions a) and b) hold. Next, assume $\tilde{A}=\operatorname{diag}\left[X_{1}, \ldots, X_{r}, A^{\prime}\right]$ and $\tilde{B}=\operatorname{diag}\left[Y_{1}, \ldots, Y_{r}, B^{\prime}\right]$; clearly that due to lemma 12 , the block structure of $\tilde{A}$ and $\tilde{B}$ is identical. There exist invertible matrices $C=\left[C_{i j}\right]$ and $D=\left[D_{i j}\right]$, $i, j \in\{1, \ldots, r+1\}$ such that $\tilde{A} C=D \tilde{B}$. The last equality can be expanded as follows: 


$$
\left[\begin{array}{cccc}
X_{1} C_{11} & \ldots & X_{1} C_{1 r} & X_{1} C_{1, r+1} \\
\vdots & \ddots & \vdots & \vdots \\
X_{r} C_{r 1} & \ldots & X_{r} C_{r r} & X_{1} C_{r, r+1} \\
A^{\prime} C_{r+1,1} & \ldots & A^{\prime} C_{r+1, r} & A^{\prime} C_{r+1, r+1}
\end{array}\right]=\left[\begin{array}{cccc}
D_{11} Y_{1} & \ldots & D_{1 r} Y_{r} & D_{1, r+1} B^{\prime} \\
\vdots & \ddots & \vdots & \vdots \\
D_{r 1} Y_{1} & \ldots & D_{r r} Y_{r} & D_{r, r+1} B^{\prime} \\
D_{r+1,1} Y_{1} & \ldots & D_{r+1, r} Y_{r} & D_{r+1, r+1} B^{\prime}
\end{array}\right]
$$

Since $A^{\prime}$ and $B^{\prime}$ are $J^{2}$-matrices and due to lemma 13 , matrix equality above implies that $C_{1, r+1} \equiv C_{2, r+1} \equiv \cdots \equiv C_{r, r+1} \equiv 0(\bmod J)$ and $D_{r+1,1} \equiv D_{r+1,2} \equiv \cdots \equiv D_{r+1, r} \equiv 0$ $(\bmod J)$. But that means that $C_{r+1, r+1}$ and $D_{r+1, r+1}$ are invertible and therefore $A^{\prime} \sim B^{\prime}$.

Conversely, assume that a), b) and c) are true. Without loss of generality, if the fundamental forms contain $S$-blocks, one may assume that blocks $X_{i}$ and $Y_{i}$ can be (independently) rearranged in such a way that starting with some positive integer $s$, all $X_{i}$ and $Y_{i}$ are $S$-blocks and $X_{i} \triangleq Y_{i}$, for all $s \leqslant i \leqslant r$. According to lemma 11 and condition $\mathrm{b}$ ) of the current lemma, there exist invertible matrices $C_{i}$ and $D_{i}$ such that $X_{i} C_{i}=D_{i} Y_{i}, s \leqslant i \leqslant r$. Due to condition c) there exist such invertible $C_{r+1}, D_{r+1}$ that $A^{\prime} C_{r+1}=D_{r+1} B^{\prime}$. Set

$$
C=\operatorname{diag}\left[I_{m_{a}}, C_{s}, \ldots, C_{r}, C_{r+1}\right]
$$

and

$$
D=\operatorname{diag}\left[I_{m_{b}}, D_{s}, \ldots, D_{r}, D_{r+1}\right]
$$

where $m_{a}$ equals total number of columns in all blocks $X_{i}, 1 \leqslant i \leqslant s-1$ and $m_{b}$ is the total number of rows in all blocks $Y_{i}, 1 \leqslant i \leqslant s-1$. If the fundamental forms do not contain $S$-blocks then

$$
C=\operatorname{diag}\left[I_{m_{a}}, C_{r+1}\right]
$$

and

$$
D=\operatorname{diag}\left[I_{m_{b}}, D_{r+1}\right]
$$

where $m_{a}$ equals total number of columns in all blocks $X_{i}, 1 \leqslant i \leqslant r$ and $m_{b}$ is the total number of rows in all blocks $Y_{i}, 1 \leqslant i \leqslant r$.

Then clearly $C$ and $D$ are invertible and $\tilde{A} C=D \tilde{B}$ which in turn implies that $A \sim B$.

To simplify the formulations in the next section, it will be useful to capture an important concept that emerged in lemma 14

Definition. If condition $b)$ of lemma 14 holds for matrices $\tilde{A}$ and $\tilde{B}$, the multisets $\subseteq(\tilde{A})$ and $\circlearrowleft(\tilde{B})$ are called affined and the relationship is denoted as $\subseteq(\tilde{A}) \triangleq \Im(\tilde{A})$.

Finally, matrices over $J^{2}$ have the following properties with respect to equivalence.

Lemma 15. Every $J^{2}$-matrix $A$ is equivalent to a matrix of the form:

$$
\left[\begin{array}{cc}
I_{m} u v & 0 \\
0 & 0
\end{array}\right]
$$


Two $J^{2}$-matrices $A$ and $B$ are equivalent iff their representation 7 has the same dimensions of the identity matrix.

Proof. The proof directly follows from lemma 2 remembering that $J^{3}=0$ and therefore $J^{2}$, as an $L$-module, is isomorphic to a single instance of $F$, thus reducing the question of equivalence of $J^{2}$-matrices to equivalence of matrices over field $F$.

\section{Canonical Form and Proof of the Main Theorem}

At this point a more extensive formulation of the main result can be provided.

Theorem. (Expanded version) Let $L$ be a local ring with two-generated maximal ideal $J=$ $L u+L v$ such that $u^{2}=v^{2}=0$ and $u v \neq 0$. Then the problem of matrix equivalence over $L$ is tame. Every matrix A over $L$ is equivalent to a canonical matrix of the form:

$$
\left[\begin{array}{cccc}
I_{n} & 0 & 0 & 0 \\
0 & X & 0 & 0 \\
0 & 0 & N_{m} & 0 \\
0 & 0 & 0 & 0
\end{array}\right]
$$

where $X$ is a block-diagonal matrix the diagonal blocks of which are of (some or all of the) $P, Q, R$ and $S$-types, $N_{m}=I_{m} u v$. Two matrices $A$ and B with corresponding canonical matrices

$$
\left[\begin{array}{cccc}
I_{n_{A}} & 0 & 0 & 0 \\
0 & X_{A} & 0 & 0 \\
0 & 0 & N_{m_{A}} & 0 \\
0 & 0 & 0 & 0
\end{array}\right]
$$

and

$$
\left[\begin{array}{cccc}
I_{n_{B}} & 0 & 0 & 0 \\
0 & X_{B} & 0 & 0 \\
0 & 0 & N_{m_{B}} & 0 \\
0 & 0 & 0 & 0_{B}
\end{array}\right]
$$

are equivalent iff the following conditions take place:

a) $n_{A}=n_{B}, \operatorname{dim} X_{A}=\operatorname{dim} X_{B}, m_{A}=m_{B}$ and $\operatorname{dim} 0_{A}=\operatorname{dim} 0_{B}$

b) $\mathfrak{P}\left(X_{A}\right)=\mathfrak{P}\left(X_{B}\right), \mathfrak{Q}\left(X_{A}\right)=\mathfrak{Q}\left(X_{B}\right), \mathfrak{R}\left(X_{A}\right)=\mathfrak{A}\left(X_{B}\right)$ and $\mathfrak{S}\left(X_{A}\right) \triangleq \mathfrak{S}\left(X_{B}\right)$

Proof. The proof directly follows from lemmas 1 in [4], 14 and 15. 


\section{References}

[1] C. W. Curtis, I. Reiner, Representation Theory of Finite Groups and Associative Algebras, AMS Chelsea Pub, 2006.

[2] P. M. Gudivok, On the Equivalence of Matrices over Commutative Rings, Infinite groups and related algebraic structures, Akad. Nauk Ukrainy, Inst. Mat., Kiev, 1993, pp. 431437.

[3] A. Yakyma, On the Equivalence of Matrices over Commutative Rings, Visnyk UzhNU, Uzhgorod, 2002, pp. 120-125.

[4] A. Yakyma, Matrix Equivalence and Reduction to Canonical Form over Commutative Artinian Rings with 2-nilpotent Jacobson Radical Research Gate, DOI: 10.13140/RG.2.2.29149.67044, August 2018.

[5] F. R. Gantmacher, Theory of Matrices, Vol. 2, AMS Chelsea Pub, 1959. 